\title{
AVALIAÇÃO DA QUALIDADE POSICIONAL DE ORTOIMAGENS RAPIDEYE
}

\author{
Rafael D. Ferreira, Leonardo S. Loureiro Silva, Aline L. Coelho, \\ Marcelo Rodrigues de A. Maranhão, Leila F. de Oliveira e Renan de A. Soares
}

Instituto Brasileiro de Geografia e Estatística

\begin{abstract}
Resumo
Imagens de sensoriamento remoto são amplamente utilizadas na extração de informações para a geração de produtos cartográficos, devendo ser avaliadas quanto à sua qualidade posicional. O objetivo do trabalho foi avaliar a qualidade posicional de um lote de ortoimagens RapidEye em uma porção do território brasileiro e verificar a possibilidade de utilização dos dados de propriedades rurais georreferenciadas do SIGEF/INCRA no processo de avaliação. Os resultados indicaram a existência de ortoimagens RapidEye com características planimétricas não correspondentes às especificações do produto, sendo observada a ocorrência de discrepâncias espacialmente concentradas. Foi possível concluir que os dados georreferenciados de propriedades rurais disponibilizados pelo SIGEF são passíveis de utilização na avaliação da qualidade posicional de imagens.
\end{abstract}

Palavras-chave: exatidão cartográfica; propriedades rurais georreferenciadas; pontos de controle; SIGEF.

\begin{abstract}
Remote sensing images have been widely used in feature extraction for cartographic production and should be evaluated as to their positional quality. This study aims to evaluate the positional quality of a batch of RapidEye orthoimages in a defined area of Brazil and verify the usability of SIGEF/INCRA's georeferenced rural properties data in the evaluation process. The results indicated the existence of RapidEye orthoimages with planimetric characteristics out of product specifications and the occurrence of spatially concentrated discrepancies. It was possible to conclude that georeferenced rural properties data provided by SIGEF are able to be used in assessing the positional quality of images.
\end{abstract}

Key words: cartographic accuracy; georeferenced rural properties; ground control points; SIGEF.

A geração de produtos cartográficos através da utilização de insumos de sensoriamento remoto mostra-se fundamental em um país de dimensões continentais como o Brasil. As imagens orbitais, por exemplo, permitem o recobrimento sistemático de áreas extensas e remotas, com informações cada vez mais detalhadas sobre a superfície terrestre. A extração de informações dessas imagens consiste, basicamente, no processo de identificar e delimitar os elementos de interesse na superfície terrestre, de acordo com a escala de mapeamento.

Para garantir a assertividade das feições mapeadas é necessário que a extração das mesmas seja realizada a partir de bases confiáveis, ou seja, produtos com acurácia posicional adequada. Nesse sentido, imagens ortorretificadas podem ser utilizadas como referência, pois são submetidas à correções das distorções devido à geometria de imageamento e ao relevo. Entretan- 
to, assim como qualquer produto cartográfico, as ortoimagens também apresentam incertezas quanto à realidade representada.

No processo de avaliação da qualidade das ortoimagens são utilizados pontos de verificação que podem ser coletados em campo ou a partir de outro produto cartográfico de melhor qualidade geométrica. Após a identificação dos pontos de verificação e as feições correspondentes nas ortoimagens, calcula-se as estatísticas das diferenças posicionais entre ambos, estabelecendo então a sua acurácia posicional.

Nos últimos anos, diversos trabalhos avaliaram a qualidade planimétrica de imagens orbitais de alta resolução espacial, indicando a possibilidade de utilização das mesmas em mapeamentos (AGUILAR et al., 2006; DAVIS \& WANG, 2003). Antunes e Siqueira (2013) avaliaram a qualidade planimétrica de ortoimagens RapidEye no município de Seropédica-RJ através da utilização de ortofotomosaicos produzidos pelo IBGE.

Em novembro de 2012, o Ministério do Meio Ambiente adquiriu uma coleção de imagens ortorretificadas do satélite RapidEye, captadas durante o ano de 2011 sobre todo o território brasileiro. De acordo com as especificações técnicas do produto, as imagens deveriam apresentar precisão planimétrica compatível com a escala 1:50.000 (Padrão de Exatidão Cartográfica - PEC, Classe A). O IBGE dispõe de dados adequados para avaliar a qualidade posicional de ortoimagens, todavia, estes dados de referência cobrem o território de forma parcial, dificultando o procedimento de avaliação em algumas regiões do país. Nesse contexto, a utilização das coordenadas dos vértices das propriedades rurais georreferenciadas do Brasil, disponibilizadas pelo Sistema de Gestão Fundiária (SIGEF) do INCRA, surge como alternativa.

O objetivo deste trabalho é avaliar a qualidade posicional de ortoimagens RapidEye adquiridas pelo Ministério do Meio Ambiente (cobertura 2011) em uma porção do território brasileiro. Propõe-se, ainda, verificar a possibilidade de utilização dos dados georreferenciados das propriedades rurais certificadas do SIGEF no processo de avaliação da acurácia posicional das ortoimagens.

\section{Materiais e Métodos}

\section{Dados de referência para a avaliação}

Nos últimos anos, o IBGE determinou, em campo, as coordenadas de diversos pontos de controle identificáveis em imagens aéreas e orbitais, no intuito de serem utilizados em processos de ortorretificação, na produção do mapeamento topográfico e, também, na avaliação de insumos para o Plano Nacional de Gestão de Riscos e Resposta a Desastres Naturais (BRASIL, 2016). Embora este método seja acurado, apresenta alto custo logístico e financeiro, dificultando sua utilização em larga escala sobre o território nacional.

Uma alternativa eficaz é a utilização de produtos cartográficos com escala igual ou superior à do insumo a ser avaliado. Nesse sentido, é comum a utilização de ortofotos provenientes de levantamentos aerofotogramétricos para a avaliação da qualidade posicional de imagens de satéli- 
te de média/alta resolução. Ainda assim, considerando as dimensões do território brasileiro, diversas áreas do país não estão cobertas com dados de referência dessa natureza para a execução adequada da avaliação posicional. A Figura 1 apresenta a distribuição dos 2.686 pontos de verificação, cujas coordenadas foram utilizadas como referência para este estudo, classificadas quanto à origem da informação.

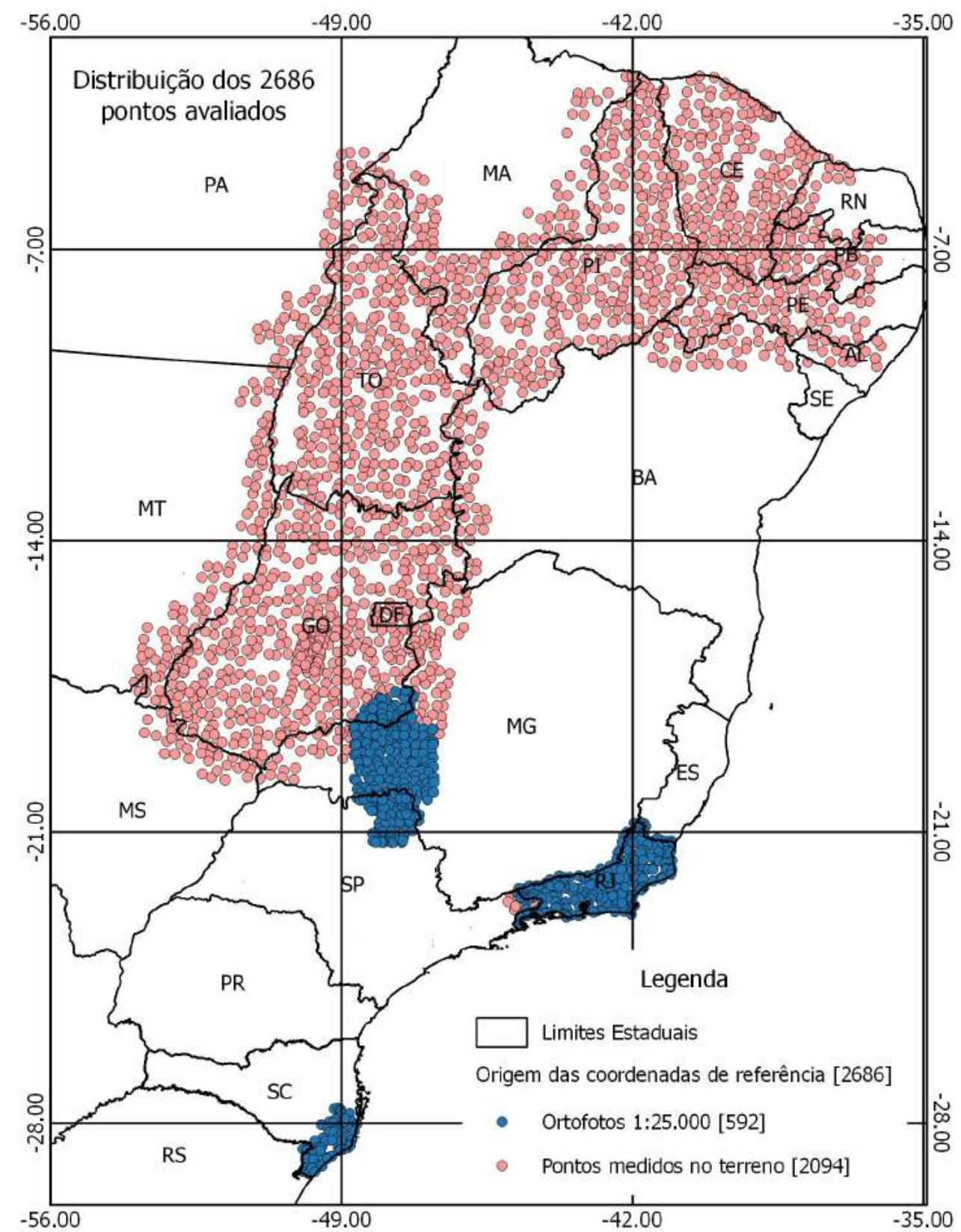

Figura 1. Classificação e distribuição dos pontos de verificação utilizados. 
Em 2013, o Sistema de Gestão Fundiária (SIGEF) entrou em operação. Desenvolvido pelo Instituto Nacional de Colonização e Reforma Agrária - INCRA, o SIGEF surgiu para modernizar a regularização de terras no país, automatizando o processo de georreferenciamento e possibilitando maior controle sobre as informações produzidas (TOLEDO \& BERTOTTI, 2014). Considerando que, de acordo com as normas vigentes, o georreferenciamento das propriedades rurais deve ser executado com a utilização de equipamentos de precisão e por mão de obra especializada (INCRA, 2015), os dados gerados podem ser uma alternativa para a avaliação da qualidade posicional de imagens em regiões com escassez de informações.

Para a realização deste trabalho foram considerados os dados classificados como "imóveis certificados privados" e "imóveis certificados SIGEF". O acesso aos dados é gratuito, através do portal do Acervo Fundiário do INCRA (INCRA, 2014). A distribuição dos imóveis georreferenciados certificados no Brasil está representada na Figura 2.

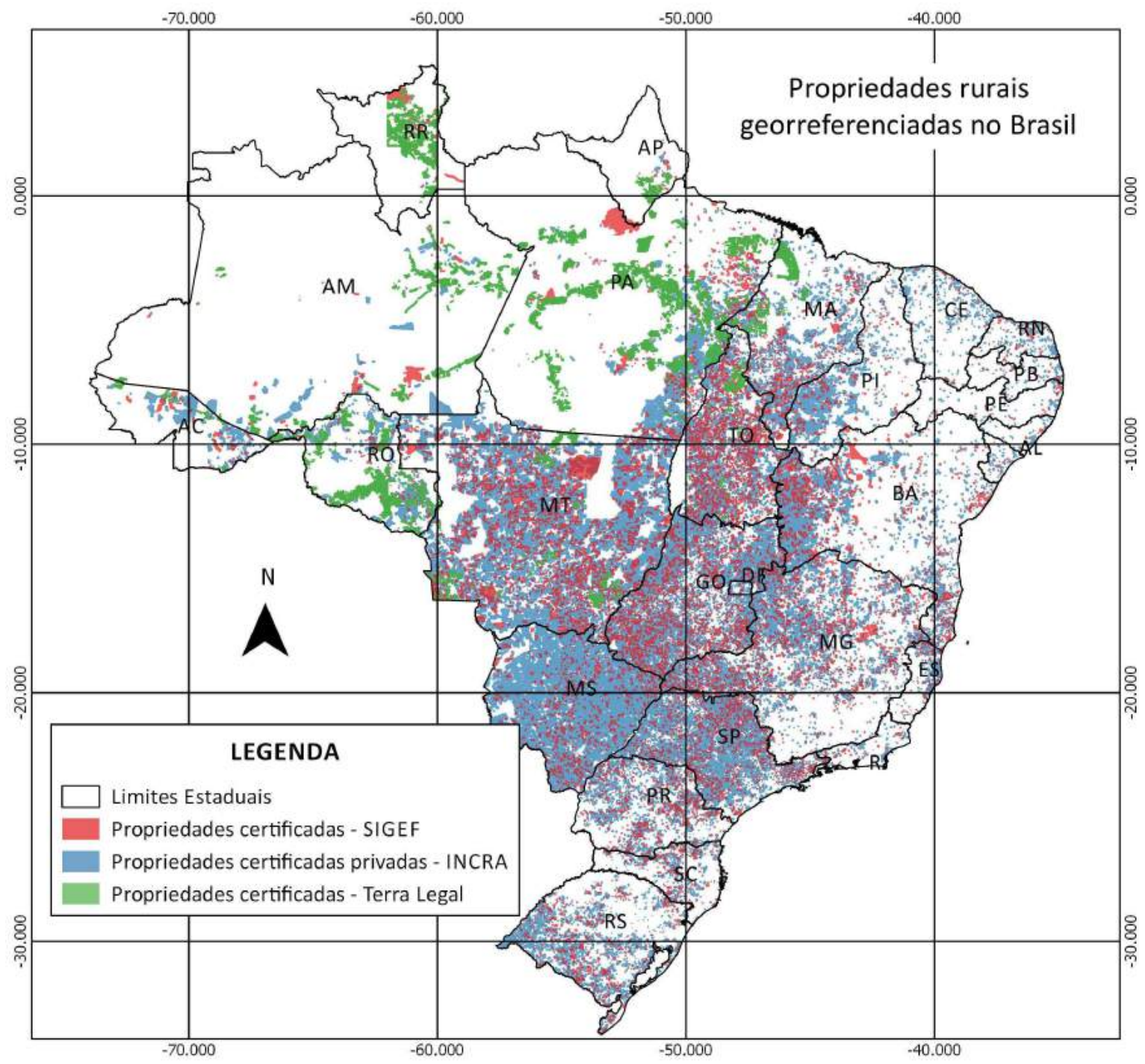

Figura 2. Distribuição das propriedades rurais georreferenciadas certificadas no Brasil, até julho de 2014. Fonte: INCRA (2014). 


\section{Ortoimagens RapidEye}

RapidEye é uma constelação composta por cinco satélites multiespectrais de cinco bandas, capazes de adquirir imagens ópticas com 77 km de largura. Após a realização de correções geométricas, imagens ortorretificadas com tamanho de pixel de 5 metros são disponibilizadas comercialmente (RAPIDEYE, 2015).

As ortoimagens avaliadas fazem parte do primeiro lote de recobrimento do território brasileiro adquirido pelo Ministério do Meio Ambiente (lote de 2011). De acordo com as especificações contratuais, as imagens deveriam ser entregues ortorretificadas, com precisão planimétrica compatível com a escala 1:50.000, PEC classe A, ou seja, não deveriam apresentar deslocamentos maiores do que 25 metros em ao menos $90 \%$ dos pontos testados.

Considerando estas especificações, foram avaliados 2.075 tiles de imagens RapidEye sobrepostos aos 2.686 pontos de verificação. Cabe ressaltar que os pontos de verificação localizados na interseção entre dois tiles, cujas datas de aquisição fossem diferentes, foram medidos duas vezes (um em cada tile). Neste caso, os pontos foram utilizados para avaliar as duas ortoimagens com sobreposição.

\section{Avaliação da qualidade posicional}

A primeira etapa da avaliação consistiu na identificação e seleção das feições homólogas entre as ortoimagens e os dados de referência (pontos de controle e feições em ortofotos), calculando-se, em seguida, os deslocamentos encontrados. Em um segundo momento, no intuito de testar a utilização dos dados georreferenciados do SIGEF como referência, foi selecionada uma pequena área na qual também houvessem pontos coletados em campo pelo IBGE. Dessa maneira, seria possível comparar as estatísticas das avaliações executadas, para os diferentes dados de referência.

De maneira geral, as feições homólogas entre as ortoimagens RapidEye e os pontos de controle coletados em campo ou feições homólogas das ortofotos, são facilmente identificáveis, considerando prioritariamente os pontos nos entroncamentos de vias. Entretanto, para os dados do SIGEF, a identificação pode ser um pouco mais complexa. Neste caso, os pontos de controle foram escolhidos após análise detalhada das poligonais das propriedades, priorizando os vértices mais representativos identificáveis nas imagens. Caso existisse dúvida pelo analista, o ponto deveria ser desconsiderado. Também foi adotada a cautela de desconsiderar os vértices em limites naturais, adjacentes a cursos d'água e linhas de cumeada, uma vez que nessas áreas a identificação fica prejudicada.

Após a escolha dos pontos foi calculado o deslocamento planimétrico resultante $\left(\Delta p_{i}\right)$ para cada ponto de verificação, conforme a equação a seguir:

$$
\Delta p_{i}=\sqrt{\Delta E_{i}^{2}+\Delta N_{i}^{2}}
$$


onde:

$\Delta E_{i}=$ coordenada $E$ coletada na ortoimagem RapidEye menos a coordenada $E$ de referência ( $E_{\text {orto- }}$ imagem ${ }^{-} E_{\text {referência }}$ ), para cada ponto;

$\Delta N_{i}=$ coordenada $N$ coletada na ortoimagem RapidEye menos a coordenada $N$ de referência $\left(N_{\text {or- }}\right.$ toimagem- $\mathrm{N}_{\text {referência), }}$, para cada ponto.

A avaliação da qualidade posicional das ortoimagens é realizada para verificar a conformidade com as Instruções Reguladoras das Normas Técnicas da Cartografia Nacional, estabelecidas no Decreto-Lei nº 89.817 de 1984, que define as tolerâncias para o PEC (BRASIL, 1984). O Decreto-Lei estabelece ainda que o PEC é um indicador estatístico de dispersão, relativo a 90\% de probabilidade, que define a acurácia de produtos cartográficos. Esta probabilidade de $90 \%$ corresponde a 1,6449 vezes o erro padrão, também denominado de erro quadrático médio (EQM) pelas Instruções (BRASIL, 1984). A Tabela 1 apresenta as tolerâncias para os produtos na escala 1:50.000, divididas entre as três classes estabelecidas no Decreto-Lei supracitado.

Tabela 1. Padrão de exatidão cartográfica para a escala 1:50.000

\begin{tabular}{ccc}
\hline \multirow{2}{*}{ Classe } & \multicolumn{2}{|c}{ Tolerância Planimétrica } \\
\cline { 2 - 3 } & PEC $(\mathrm{m})$ & EQM \\
\hline A & 25 & 15 \\
B & 40 & 25 \\
C & 50 & 30
\end{tabular}

\section{Resultados e Discussão}

A distribuição dos pontos utilizados na primeira análise (somente pontos levantados em campo e em ortofotos) e os respectivos deslocamentos planimétricos resultantes estão apresentados na Figura 3.

Analisando a Figura 3 é possível observar que os maiores deslocamentos estão concentrados espacialmente na porção oeste do Estado de Minas Gerais. Considerando os deslocamentos planimétricos resultantes de todos os pontos analisados, verificou-se que aproximadamente $30 \%$ apresentaram deslocamentos maiores que 25 metros. Por outro lado, aproximadamente $70 \%$ dos tiles avaliados atenderam ao PEC Classe A para a escala 1:50.000.

Os tiles de ortoimagens RapidEye com maior deslocamento posicional, concentrados na região oeste de Minas Gerais, foram reavaliados, utilizando os dados do SIGEF como referência. Os resultados obtidos nesta segunda avaliação foram comparados com 98 pontos críticos, correspondentes aos mesmos tiles, selecionados a partir da primeira avaliação e apresentados na Tabela 2. 


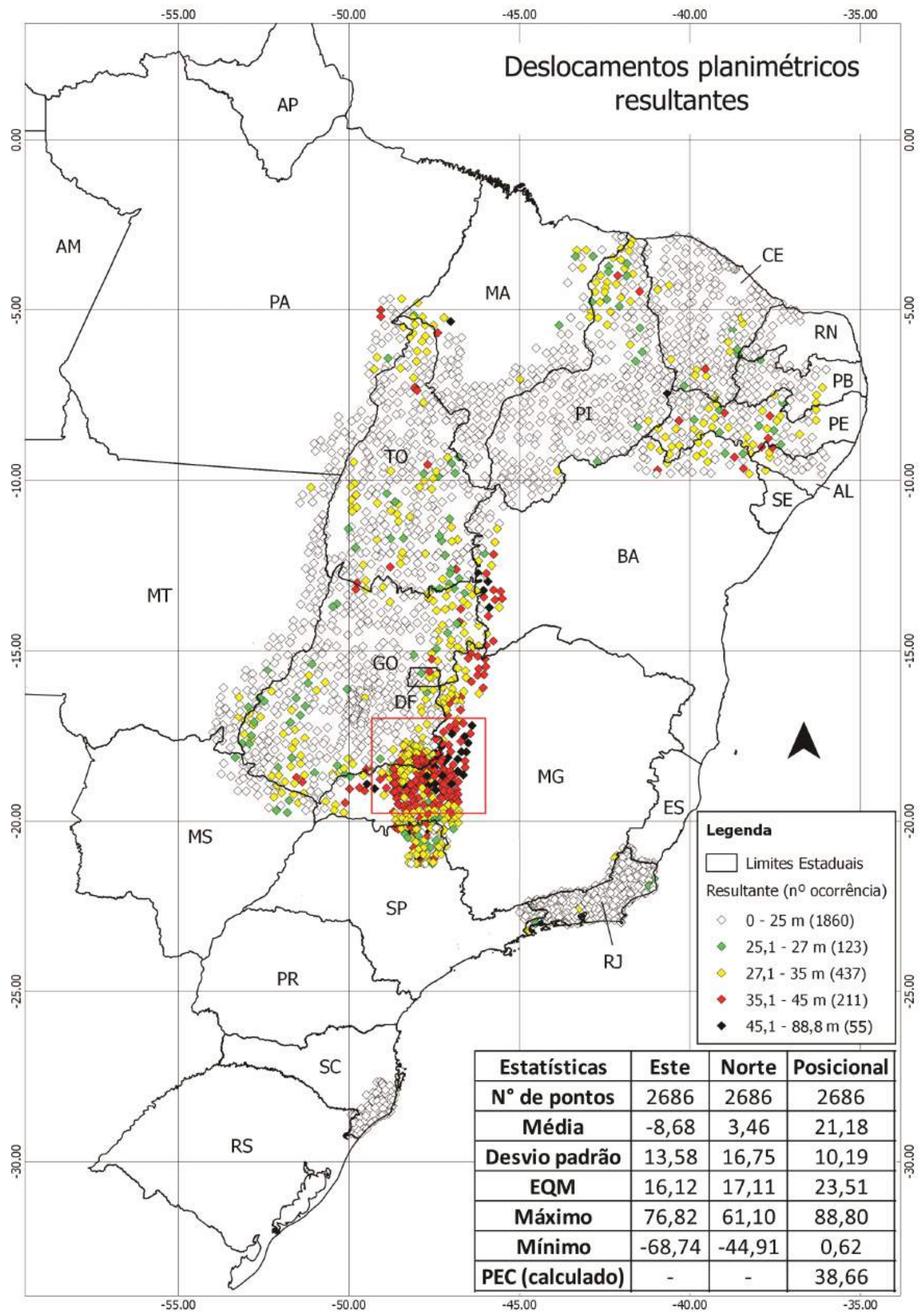

Figura 3. Deslocamento planimétrico resultante, com destaque para a maior concentração de deslocamentos no Estado de Minas Gerais, e estatísticas dos pontos avaliados. PEC (Calculado)=EQM^1,6449. 
Tabela 2. Comparação entre as estatísticas posicionais geradas pela utilização de diferentes dados de referência (SIGEF x Pontos de campo). PEC (Calculado)=EQM¹,6449.

\begin{tabular}{l|rrrr|rrr}
\hline \multirow{2}{*}{ Estatísticas } & \multicolumn{3}{c|}{ Pontos SIGEF - INCRA/MDA } & \multicolumn{3}{c}{ Pontos de campo - IBGE } \\
\cline { 2 - 8 } & \multicolumn{1}{|c|}{ Este } & \multicolumn{1}{c}{ Norte } & Resultante & \multicolumn{1}{c}{ Este } & Norte & Resultante \\
\hline Número de pontos & 318 & 318 & 318 & 98 & 98 & 98 \\
Média & 26,83 & 30,86 & 42,37 & 24,17 & 31,70 & 40,79 \\
Desvio padrão & 11,30 & 10,41 & 10,51 & 13,96 & 6,07 & 9,09 \\
EQM & 29,12 & 32,61 & 43,72 & 26,66 & 32,44 & 41,99 \\
Máximo & 2,73 & 68,64 & 84,83 & 76,82 & 48,91 & 88,80 \\
Mínimo & $-58,13$ & 0,42 & 18,00 & $-44,40$ & 17,30 & 18,89 \\
PEC (calculado) & - & - & 71,92 & - & - & 69,07
\end{tabular}

A utilização dos dados do SIGEF aumentou o número de amostras disponíveis para a avaliação da qualidade posicional das imagens. Entretanto, embora o número amostral, considerando os pontos do SIGEF, seja cerca de três vezes maior do que as amostras da primeira análise, os resultados são estatisticamente condizentes. Os valores de média, desvio padrão e EQM foram coerentes utilizando ambos os conjuntos de dados de referência. Dessa forma, o PEC (calculado) foi consistente, garantindo a mesma classificação dos produtos testados, independentemente da metodologia utilizada.

\section{Conclusões}

Através do presente estudo foram detectadas ortoimagens RapidEye com características planimétricas fora das especificações previstas no contrato de aquisição das mesmas, totalizando cerca de $30 \%$ das ortoimagens avaliadas. A maior parte dessas ortoimagens está concentrada na porção oeste do Estado de Minas Gerais. É importante ressaltar que as imagens da coleção de 2011 foram reprocessadas e substituídas pelo fornecedor, com a devida correção dos deslocamentos identificados neste trabalho.

A avaliação realizada neste estudo indica que os dados georreferenciados de propriedades rurais disponibilizados pelo SIGEF/INCRA podem ser utilizados para avaliar a qualidade posicional de imagens na escala 1:50.000. Para o conjunto amostral utilizado, os valores de média, desvio padrão e EQM das discrepâncias calculados com base nos vértices de poligonais do SIGEF foram compatíveis com aqueles obtidos nos testes realizados com os pontos determinados em campo pelo IBGE.

Dados georreferenciados levantados em campo, disponibilizados de forma gratuita no portal do SIGEF na internet, podem ser utilizados para a redução de gastos com missões para a coleta dessas informações. Além disso, a utilização dos dados do SIGEF pode incrementar o número de pontos de verificação em regiões onde os dados de referência são escassos. 
Manuscrito recebido para publicação em janeiro de 2016. 
Referências

AGUILAR, M.A.; AGUILAR, F.J.; CARVAJAL, F.; AGUERA, F.; ESTRADA, L. Geometric accuracy of IKONOS geo panchromatic orthoimage products. Revue Française de Photogrammétrie et de Télédétection v.184, p. 5-10, 2006.

ANTUNES, M.A.H.; SIQUEIRA, J.C.S. Características das imagens RapidEye para mapeamento e monitoramento e agrícola e ambiental. In: SIMPÓSIO BRASILEIRO DE SENSORIAMENTO REMOTO, 16., São José dos Campos. Anais... São José dos Campos: INPE, 2013, p. 547554.

BRASIL. Decreto-lei $n^{0} 89.817$ de 20 de junho de 1984. Estabelece as Instruções Reguladoras das Normas Técnicas da Cartografia Nacional. Diário Oficial da União, Brasília, 1984.

BRASIL. Lei $n^{\circ} 13.249$ de 13 de janeiro de 2016. Institui o Plano Plurianual da União para o período de 2016 a 2019. Diário Oficial da União, Brasília, 2016.

DAVIS, C.H.; WANG, X. Planimetric accuracy of Ikonos $1 \mathrm{~m}$ panchromatic orthoimage products and their utility for local government GIS basemap applications. International Journal of Remote Sensing v.24, n.22, p. 4267-4288, 2003.

INCRA. Acervo Fundiário: INCRA. Disponível em:<http://acervofundiario.incra.gov.br/>. Acesso em jul. 2014.

INCRA. Manual do SIGEF. Disponível em:<sigef.incra.gov.br>. Acesso em nov. 2015.

RAPIDEYE. Satellite Imagery Product Specifications. Disponível em:<blackbridge.com/rapideye/>. Acesso em 12 de nov. 2015.

TOLEDO, B.H.C.; BERTOTTI, L.G. Breve histórico da certificação de imóveis rurais no Brasil e apresentação do Sistema de Gestão Fundiária - SIGEF. Ambiência v.10, n.3, p. 839-847, 2014. 